\title{
Transvesical Colorectal Anastomosis: An Unusual Complication inReversal of Hartmann's Procedure
}

Fabio Pacifico*, Pier Paolo Vergineo, Paolo Ferravante and Rodolfo Vincenti

Ospedale Fatebenefratelli Surgery Viale Principe di Napoli 14/A Benevento, BN 82100, Italy

\begin{abstract}
It is described an unusual complication after colorectal surgery for reversal of Hartmann's procedure. The female patient had been operated on for diverticulitis (Hinchey III) according to Hartmann's procedure. Six months later she underwent restorative surgery with circular stapler for end-to-end transanal anastomosis. The onset of symptoms such as fever, abdominal pain, fecaluria and pneumaturia had forced the patient to a hospital readmission. Diagnostic examinations had revealed colovesical fistula. The cause of this complication was that staple line of colorectal anastomosis had incorporated the bladder wall to full thickness. It was necessary to reoperation for the resolution of the damage. The resection of fistulised tract, the colorectal reanastomosis and the reconstruction of the bladder were performed.
\end{abstract}

Keywords: Hartmann's procedure; Transanal anastomosis

\section{Introduction}

The reestablishment of intestinal continuity after Hartmann's procedure, with conventional surgery or laparoscopy, is burdened with a complication's rate ranging from $13 \%$ to $50 \%$ of cases [1-3]. Because of these data, the reversal is restricted to a low number of patients, slightly more than $1 / 3$, in good clinical condition (lower ASA) and not too old. The most frequent complication is represented by infection with average rate equal to $12.5 \%$ [2]. A very rare chance of inclusion in the anastomosis of the bladder wall for lack of recognition with a consequent rectovesical fistula is very rare. We describe the case of an unusual fistula between colorectal anastomosis and bladder occurred in a female patient. Correction with bladder reconstruction and end toend colorectal reanastomosis were done. Probably this case is the first described in the literature.

\section{Clinic Case}

A 78-year-old female patient with a history of complicated diverticular disease is reported. About 10 months before the patient surgically had been treated for perforated sigmoid diverticulum (Hinchey III). The anterior resection and left colostomy according to Hartmann's procedure were done. After about 6 weeks she was re-operated for intestinal obstruction due to internal adhesions and ileal loop. After an additional 6 months she was surgically treated for reversal of Hartmann's procedure and incisional hernia by the same surgeon. Three months later appeared fever, abdominal pain, fecaluria, pneumaturia and the patient comes to our observation. The cystography highlights the abnormal filling of the bladder with the metal clips to the center of the colo-rectal anastomosis, (Figure 1a) and a mild filtering of the contrast medium. Figure $1 \mathrm{~b}$ a subsequent $\mathrm{CT}$ scan shows the portion of the colon anastomosed to bring "knight" of the bladder dome and fistula sinking into the bladder at the anastomotic colorectal ring (Figure 2a, 2b, 2c, 2d). Therefore it was decided to refer the patient to a new surgery with the diagnosis of colovesical fistula. The surgery was performed with a new median laparotomy. It was required a difficult adhesiolysis for the identification of anatomical structures in the pelvic area. It is isolated the colon until the end bladder wall. The distension of the bladder with saline solution shows a "donut" shape surrounding the colonic stump (Figure 3). It attacks the rectum under the bladder that seems to penetrate into the bladder. Cystoscopy confirms this finding of colo-rectal transvescical anastomosis (Figure 4), therefore proceeds to longitudinal opening of the bladder, releasing the intestinal tract. Is then reconstructed the bladder wall in a single layer resorbable and a sovrapubic bladder drainage was leaved. After resection of the fistulized intestinal tract we have performed a colorectal reanastomosis transanal end-to-end with a circular stapler to $3.1 \mathrm{~cm}$. A "ghost ileostomy" was preferred. The postoperative course was good and uneventful. The patient was discharged on $7^{\text {th }}$ postoperative day. Further complications at a mean follow up of 12 months not were found.

\section{Discussion}

The complication rate after bowel resection surgery according to Hartmann is still very high [4]. The laparoscopic technique, although in selected cases, seems to have reduced this percentage, but has a high conversion rate, more than $20 \%$ bound to numerous adhesions

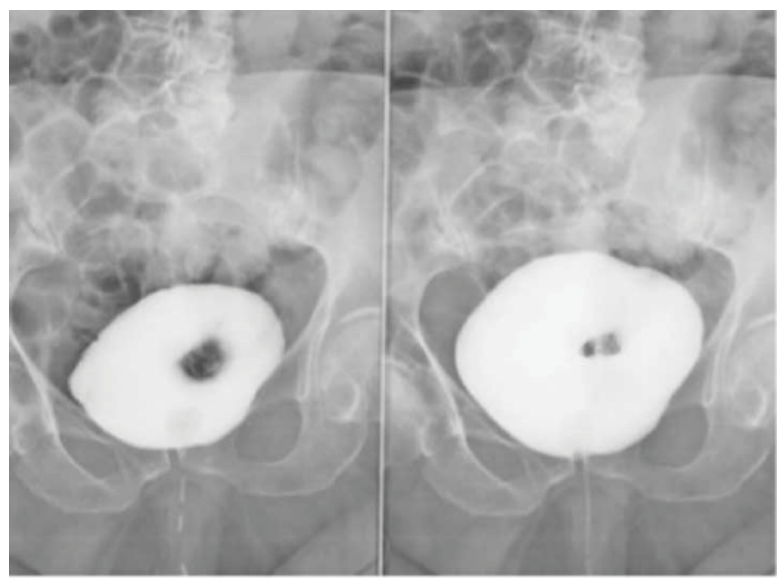

Figure 1a: Rx cistography shows anastomotic colo-rectal ring in to the bladder. 1b. A mild filtering of the contrast medium.

*Corresponding author: Pacifico F, MD, Ospedale Fatebenefratelli Surgery Viale Principe di Napoli 14/A Benevento, BN 82100, Italy, Tel: +39-824-771-223; E-mail: fabiopacifico@libero.it

Received February 02, 2015; Accepted March 28, 2015; Published April 07, 2015

Citation: Pacifico F, Vergineo PP, Ferravante P, Vincenti R (2015) Transvesical Colorectal Anastomosis: An Unusual Complication in Reversal of Hartmann's Procedure. Surgery Curr Res 5: 227. doi:10.4172/2161-1076.1000227

Copyright: @ 2015 Pacifico F, et al. This is an open-access article distributed under the terms of the Creative Commons Attribution License, which permits unrestricted use, distribution, and reproduction in any medium, provided the original author and source are credited. 
Citation: Pacifico F, Vergineo PP, Ferravante P, Vincenti R (2015) Transvesical Colorectal Anastomosis: An Unusual Complication in Reversal of Hartmann's Procedure. Surgery Curr Res 5: 227. doi:10.4172/2161-1076.1000227

and the difficulty of finding the rectal stump [1,2]. Therefore, both the conventional and laparoscopic procedure conceal pitfalls and are considered major surgery interventions who. A recent review reported complications mostly found in open surgery than in laparoscopic surgery [2]. These are cases of infection of the abdominal wall, dehiscence of the anastomosis, postoperative bleeding, anastomotic stenoses and cardiopulmonary. The reversal of Hartmann's procedure is also burdened with varying percentages of intra-operative complications. A recent study conducted on an Asian population highlights the surgical difficulties due to the previous surgery for the formation of peritoneal and visceral adhesions [5]. Point out, in fact, that even with a careful and meticulous dissection can cause intestinal lesions and/or ureteral injury in varying percentages up to $16 \%$ of cases [5]. The colorectal transvesical anastomosis is very unusual occurrence. The inclusion in circular stapler of bladder wall to full thickness does appear to have been never reported in the literature. They can occur in extreme

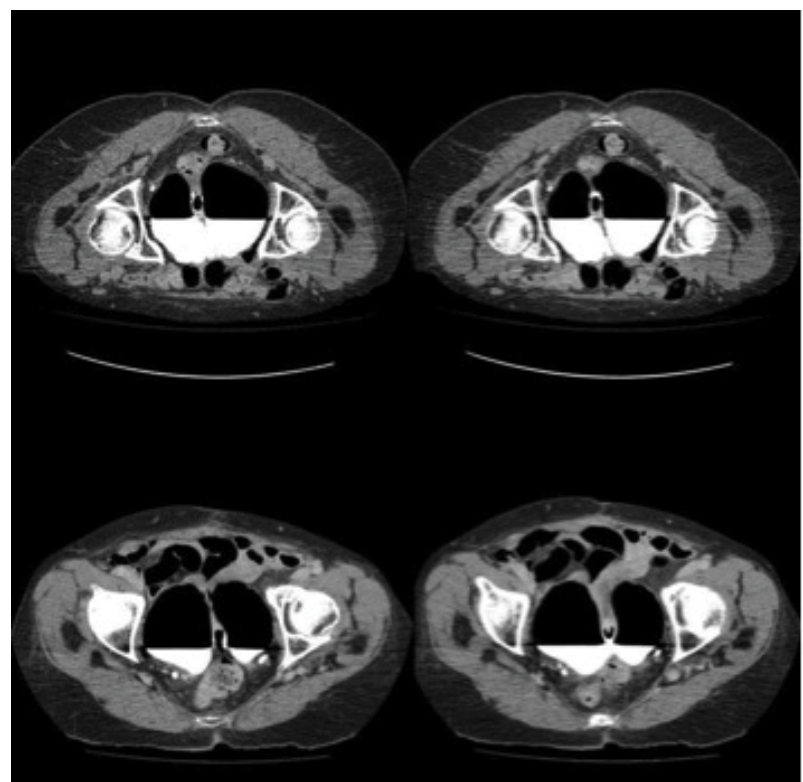

Figure 2a, 2b, 2c, 2d: TC shows the unusual passage of colon into the bladder to full thickness and the anatomotic colorectal fistula. Axial images shows a bladder divided into two parts.

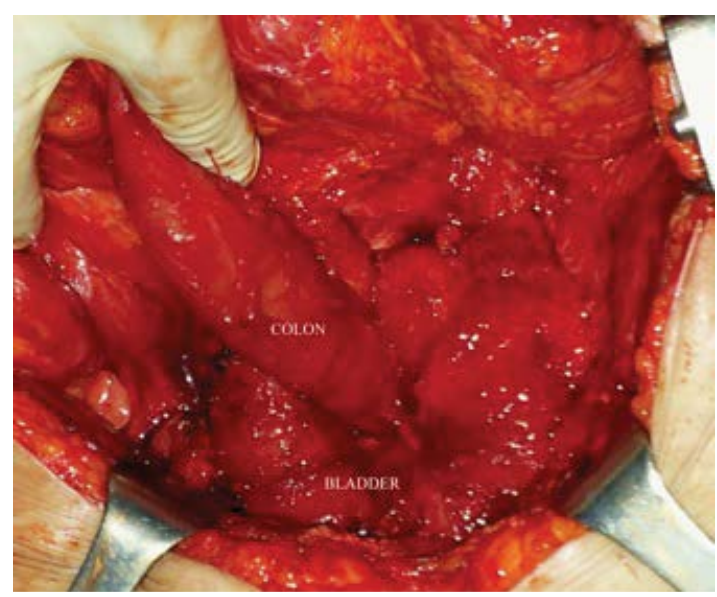

Figure 3: Intraoperative picture that shows colon through the bladder wall with suggestive "donut" shape of bladder.

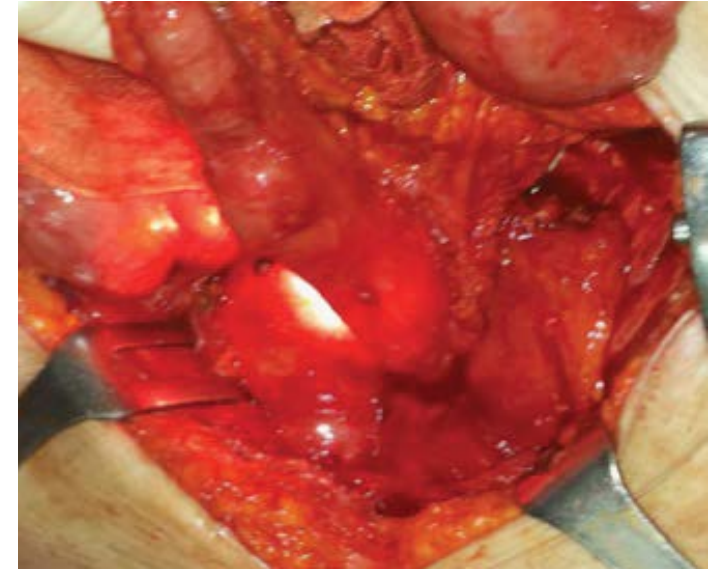

Figure 4: The same picture of previous figure showing the light of the cystoscope, which confirms transbladder anastomosis.

cases and in patients with a past history of hysterectomy. Adhesions particularly difficult do not allow the recognition of the floors and in particular bladder dome that can be pulled downward in patient with hysterectomy. Clearly this is an operator's error. The preparation of rectal remnant stump is fundamental in this procedure. The identification of stump may be easier from the insertion of rectoscope through anus. Its isolation from pelvic structures must be meticulous and a retractor could be help certainly. The timing of reversal is still discussed. In our case the restorative procedure was performed after 24 weeks. In literature there is no absolute answer of the time of reversal. Recent work has reported the possibility of such an intervention within one month in order to have smaller and looser adhesions [6]. However others emphasize the passage of at least 15 weeks [7]. In any case, the opening procedure, regardless of the timing is not without its difficulties and full of potential complications intra-operative and postoperative care. Probably in this case we think that the error was due to two factors. The rectal' stump not properly prepared and therefore not separated from the bladder's floor and the previous hysterectomy has determined a complication of all unusual. In this mode the insertion of the stapler through the anus may damage the pelvic structures. The anastomosis has incorporated the bladder to full thickness to determining the typical form of a "donut". The fistula appeared after 3 months postoperatively with abdominal pain, fecaluria, pneumaturia and fever. The reoperation was necessary to correct the damage. Were performed the resection of the fistulised colon, the full opening with subsequent reconstruction of the bladder's wall and the reanastomosis between colon and rectum.

\section{Conclusion}

Transvesical colorectal restorative bowel continuity is an unusual complication of mechanical anastomosis caused by the operator's error. We believe that the reversal Hartmann's procedure is not a particularly difficult surgery although associated with significant morbidity. The staplers can make the interventions easier but in many complex cases should be performed by experienced surgeons.

\section{References}

1. Huynh H, Trottier DC, Soto CM, Moloo H, Poulin EC, et al. (2011) Laparoscopic colostomy reversal after a Hartmann procedure: a prospective series, literature review and an argument against laparotomy as the primary approach. Can J Surg 54: 133-137. 
Citation: Pacifico F, Vergineo PP, Ferravante P, Vincenti R (2015) Transvesical Colorectal Anastomosis: An Unusual Complication in Reversal of Hartmann's Procedure. Surgery Curr Res 5: 227. doi:10.4172/2161-1076.1000227

2. Vandewall BJM, Draaisma WA, Schouten ES, Broeders IAMJ, Consten ECJ (2010) Conventional and Laparoscopic Reversal of the Hartmann Procedure: a Review of Literature. J Gastrointest Surg 14:743-752.

3. Vermeulen J, Coene PPLO, Van HoutNM, Van der Harst E, Gosselink MP, et al. (2009) Restoration of bowel continuity after surgery for acute perforated diverticulitis: should Hartmann's procedure be considered a one-stage procedure? Colorectal Disease 11: 619-624.

4. Antolovic D, Reissfelder C, Özkan T, Galindo L, Büchler MW, et al. (2011) Restoration of intestinal continuity after Hartmann's procedure-not a benign operation. Are there predictors for morbidity? Langenbeck's Arch Surg 396: 989-996.

5. Tan WS, Lim JF, Tang CL, EU KW (2012) Reversal of Hartmann's procedure: experience in an Asian population. Singapore Med J 53: 46-51.

6. Geoghegan JG, Rosemberg IL (1991) Experience with early anastomosis after the Hartmann procedure. Ann R COll Surg Engl 73: 80-82.

7. Keck JO, Collopy BT, Ryan PJ, Fink R, Mackay JR, et al. (1994) Reversal of Hartmann's procedure: effect of timing and technique on ease and safety. Dis Colon Rectum 37: 243-248. 